\title{
Modifications of muscle synergies and spinal maps due to absence of visual feedback in patients with unilateral vestibular disease
}

\author{
Monaco V., Martelli D., Nacci A., Fattori B., Berrettini S., Micera S., Senior Member, IEEE
}

\begin{abstract}
The present study aimed at describing the modifications of muscle synergies and spinal activity due to the absence of visual feedback, in patients affected by unilateral vestibular disease. Patients were tested both during unperturbed quite stance and walking while the activity of 7 bilateral muscles, from the leg to the trunk, were recorded for the estimation of muscle synergies and spinal activity. Results showed that during locomotion the absence of visual feedback did not significantly modify either the principal roles underlying muscle activity (i.e., synergies) or the spinal bursts. Conversely, during the upright stance, the absence of visual feedback involved a significant coupling of ankle dorsi- and plantar-flexor muscle groups with a consequent shift of the motoneuronal (MN) activity toward most caudal segments. Results revealed that the muscle synergies are able to document an increased activity of sensory-motor afferences leading a more intense role of the forward based mechanism underlying balance control in vestibular patients.
\end{abstract}

\section{INTRODUCTION}

An acute unilateral vestibular paralysis causes a vestibular tone imbalance, such that, the fast phase of the spontaneous rotational nystagmus and the initial perception of apparent body motion are directed away from the side of the lesion, and the postural reactions initiated by vestibulospinal reflexes are usually in a direction opposite to the direction of vertigo [1]. Consequently, a vestibular disorder limits the ability of patients to suitably achieve daily motor tasks, such as walking and/or keeping the upright stance, thus affecting their quality of life.

One of the main advantages of the integration of all sources of feedback (i.e., visual, vestibular and somatosensory) to the balance control is that the central nervous system (CNS) can adopt flexible combinations of these afferences, involving compensatory strategies aimed at enabling patients, with mild to moderate diseases, to walk

We acknowledge financial support from the European Commission $7^{\text {th }}$ FP (GA 225929) Project: CLONS CLOsed-loop Neural prostheses for vestibular disorders.

Monaco V. (corresponding author; P.zza Martiri della Libertà, 33, 56127 Pisa, Italy; phone: +39 050.883007; fax: +39 050.883101; e-mail: v.monaco@sssup.it), Martelli D. (e-mail: d.martelli@sssup.it), and Micera S. (e-mail: micera@sssup.it) are with The BioRobotics Institute, Scuola Superiore Sant'Anna, Pisa (I)

Nacci A. (e-mail: a.nacci@med.unipi.it), B. Fattori (e-mail: b.fattori@ent.med.unipi.it), and S. Berrettini (e-mail: s.berrettini@med.unipi.it) are with the Otolaryngology Unit, Ospedale Cisanello, Pisa (I)

Micera S., is also with the Translational Neural Engineering Laboratory, Center for Neuroprosthetics, Swiss Federal Institute of Technology, Lausanne $(\mathrm{CH})$. and keep the upright stance. Nevertheless, despite their ability to use compensatory strategies, motor performance of vestibular patients are characterized by significant deficiencies when compared to those of healthy subjects $[2$, $3]$.

The aim of this preliminary study is to describe muscle activity of patients with a unilateral vestibular impairment while walking or maintaining upright stance. In particular, we aimed at highlighting whether and how the absence of visual information modifies the principal roles underlying the activity of muscles recorded from their whole body, often named muscle synergies, and the activation of motoneuronal $(\mathrm{MN})$ pools related to $\mathrm{C} 4-\mathrm{S} 2$ spinal sections.

\section{MATERIAL AND METHODS}

\section{A. Subjects}

Four subjects with unilateral vestibular disorder of the left side ( 3 males and 1 female, $64.8 \pm 9.5$ years old, $79.0 \pm$ $10.1 \mathrm{~kg}, 1.72 \pm 0.03 \mathrm{~m}$ ) were enrolled for the study. At the time of evaluation, in the acute phase, all patients had a noncompensated labyrinth deficit with third-degree nystagmus directed towards the unaffected side. All patients in this study underwent the following tests: liminal tone audiometry, impedenzometry, measurement of spontaneous nystagmus (positional and positioning tests, with or without Frenzel glasses), and the Head Shaking Test (HST). In addition, all subjects underwent the vestibular caloric test according to Fitzgerald- Hallpike and videonystagmographic investigation with the Ulmer-Synapsys $($ System. Besides the above tests for the presence of vestibulopathy and damage to the vestibulo-ocular reflex (VOR), the vestibular-spinal reflex (VSR) was investigated by means of static stabilometry using the Balance Manager Static Systems NeuroCom. International, Inc (C).

We excluded from the study: patients with vestibular signs of involvement of the CNS, personal history of multiple sclerosis, Arnold-Chiari malformation, major psychiatric disorders, cerebrovascular disease, retrocochlear lesions, congenital hypoacusia, positional paroxysmal vertigo, and neurinoma of the acoustic nerve.

\section{B. Experimental procedures}

Experimental procedures consisted in two main tests: Orthostatic Posturography (OP) and Walking Trials (WT). Subjects carried out both tests with open eyes (OE) and closed eyes (CE). 
During the OP, patients were asked to maintain upright stance on a force platform (Balance Manager Static Systems NeuroCom. International, Inc (C) for two $120 \mathrm{~s}$ long trials, respectively with $\mathrm{OE}$ and $\mathrm{CE}$. Feet were located in accordance with the reference frame reported on the platform's surface and arms rested along the body. During OE trials, subjects were asked to look straight ahead at a visual reference point (a black dot, $3 \mathrm{~cm}$ in diameter) located $2 \mathrm{~m}$ away on the wall, at eyes height. The Antero-Posterior (AP) and Medio-Lateral (ML) components of the Centre of Pressure $(\mathrm{CoP})$ were measured for the entire duration of the session with a sampling rate of $100 \mathrm{~Hz}$.

During the WT, patients were asked to walk along a straight direction at a comfortable pace for a distance of $9 \mathrm{~m}$ with OE and CE. Subjects donned shoes provided with foot switches to record the heel strike from both feet. This test was carried out at least three times for each eye condition. Angle of veering from the center line during $\mathrm{CE}$ trials was recorded.

During both tests (e.g., OP and WT), the electromyographic (EMG) activity of 7 bilateral muscles (Erector Spinae at L2, ES, Gluteus Medius, GM, Rectus Femoris, RF, Biceps Femoris, BF, Gastrocnemius Lateralis, GL, Tibialis Anterior, TA and Middle Trapezius, MT) was recorded (NORAXON, Telemyo 2400T, V2) using bipolar $\mathrm{Ag}-\mathrm{AgCl}$ surface EMG electrodes placed on previously shaved and cleaned skin. Sampling rate was $3000 \mathrm{~Hz}$ and the gain of the amplifiers was 1000 .

\section{EMG data processing}

EMG signals recorded during both experimental tests were full wave rectified, low-pass filtered (zero-lag Butterworth filter, $4^{\text {th }}$ order, with cut off at $10 \mathrm{~Hz}$ ), and normalized in amplitude in accordance with the procedure described by Ivanenko and colleagues [4].

Data referring to WT were subdivided into gait cycles referred to the right heel strike. Data from to the first and the last three strides were discarded to avoid bias due to the transitory phases. Remaining data were time-interpolated over individual gait cycles on a time base of 200 points and averaged across all strides in order to have a representative data set for each muscle and each trial. Concerning the OP, first and last $15 \mathrm{~s}$ of the whole record were discarded to avoid bias due to the initial transitory phase and fatigue. This preliminary procedure provided a $200 \times 14$ matrix for each WT and a $270000 \times 14$ matrix for each OP test. These data sets were then post-processed for synergy extraction and estimation of spinal activity.

\section{Extraction of muscle synergies}

Each dataset was factorized using Factor Analysis with varimax rotation (FA) in order to extract primitive signals, representing the timing of activation, and weighting coefficients, representing muscle enrolment, related to each synergy. In this preliminary study, the number of retained synergies was 3 in accordance with our previous findings [5].

\section{E. Estimation of spatiotemporal MN activity}

Pre-processed EMG signals were used to map the spatiotemporal MN activity along the rostrocaudal direction related to the C4-S2 spinal segments, as described in literature $[4,5]$.

Briefly, for each spinal segment, the MN activity was estimated as the weighted summation of all normalized EMG signals related to muscles innervated by such segment [4]. Table I reports the innervation of all muscles recorded in this study. For each spinal map, the Center of Activity (CoA), a synthetic measure of the weighted level of activity of the spinal map, was also computed in accordance with the equation reported by Ivanenko and colleagues [4].

TABLE I. INNERVATION OF RECORDED MUSCLE GROUPS

\begin{tabular}{|c|c|}
\hline Innervation & Muscle groups \\
\hline C3-C4 & MT \\
\hline T2-T9 & ES \\
\hline L2-L3 & RF \\
\hline L4 & TA \\
\hline L5 & BF, TA \\
\hline S1 & GM, BF, LG, TA \\
\hline S2 & GM, BF, LG \\
\hline
\end{tabular}

\section{F. Postural Parameters}

According to the EMG pre-processing, the first and the last 15 seconds of the CoP-related record was discarded in order to reduce bias due to initial transitory effects and fatigue. Then, AP and ML components of the CoP were filtered using a digital low-pass FIR filter with a cut-off frequency at $10 \mathrm{~Hz}$ and subtracted by their mean value. Subsequently, according to Prieto and colleagues [6], the vector of resultant distances (RD) from the mean $\mathrm{CoP}$ to each of its points was calculated. In order to characterize the balance control during unperturbed quite stance, the following set of Postural Parameters (PPs) was adopted, due to their reported clinical revelance [7]:

- the mean velocity (MV) of the CoP, indicating the amount of energy spent;

- the root mean square of AP, ML, and RD time series (i.e., $\mathrm{RMS}_{\mathrm{AP}}, \mathrm{RMS}_{\mathrm{ML}}, \mathrm{RMS}_{\mathrm{RD}}$ ), which is a measurement of the amplitude variability of the $\mathrm{CoP}$ around the mean and is related to the amplitude of the corrective activities;

- the sway area (SA), which quantifies the relationship between the postural control system activities and the level of stability achieved;

- the $95 \%$ power frequency $\left(\mathrm{PF}_{95}\right)$, which estimates the frequency extent of the CoP time series, and is related to the periodicity exhibited by the postural control strategy; $\mathrm{PF}_{95}$ was calculated in the $\mathrm{AP}\left(\mathrm{PF}_{95 \mathrm{AP}}\right)$ and ML directions $\left(\mathrm{PF}_{95 \mathrm{ML}}\right)$. 


\section{G. Statistical Analysis}

PPs were compared between OE and CE by means of a ttest. Significance was set at $\alpha=0.05$.

Data analysis was performed off line by means of customized MATLAB (The MathWorks Inc., Cambridge, MA, US) scripts.

\section{RESUlts}

\section{A. PPs during quite stance and angle of veering during $W T$}

Although we did not observe any statistically significant difference in PPs between OE and CE (Table II), the absence of visual feedback involved a wider statokinesigram, reflecting more intense corrective actions of the CNS and consequent increased cost (see Table II and Figure 1). The periodicity of the postural control did not reveal any trend between OE and CE trials (Table II).

TABLE II. MEAN \pm STANDARD DEVIATION OF PPs

\begin{tabular}{|c|c|c|c|}
\hline & OE & CE & p-Value \\
\hline $\mathrm{MV}[\mathrm{cm} / \mathrm{s}]$ & $0.496 \pm 0.092$ & $0.754 \pm 0.328$ & 0.166 \\
\hline $\mathrm{RMS}_{\mathrm{AP}}[\mathrm{cm}]$ & $0.474 \pm 0.089$ & $0.528 \pm 0.118$ & 0.441 \\
\hline $\mathrm{RMS}_{\mathrm{ML}}[\mathrm{cm}]$ & $0.210 \pm 0.058$ & $0.246 \pm 0.054$ & 0.345 \\
\hline $\mathrm{RMS}_{\mathrm{RD}}[\mathrm{cm}]$ & $0.522 \pm 0.077$ & $0.584 \pm 0.118$ & 0.362 \\
\hline $\left.\mathrm{SA}^{2} / \mathrm{cm}\right]$ & $4.836 \pm 0.585$ & $8.700 \pm 5.300$ & 0.181 \\
\hline $\mathrm{PF}_{95 \mathrm{AP}}[\mathrm{Hz}]$ & $0.554 \pm 0.083$ & $0.574 \pm 0.157$ & 0.810 \\
\hline $\mathrm{PF}_{95 \mathrm{ML}}[\mathrm{Hz}]$ & $0.542 \pm 0.103$ & $0.536 \pm 0.186$ & 0.952 \\
\hline
\end{tabular}
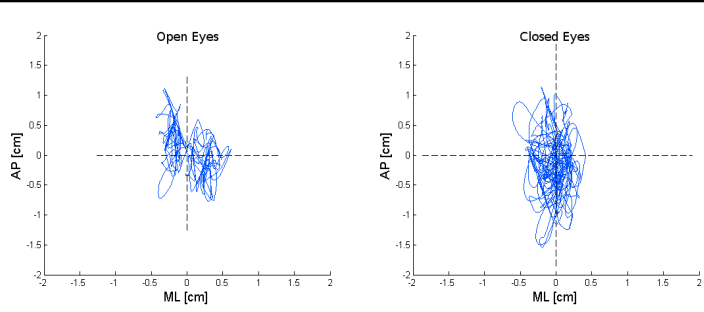

Figure 1. Representative statokinesigrams of a patient while keeping OE (on the left) and CE (on the right).

During WT with OE, all patients were able to keep the straight line. Conversely, as expected, when they walked with $\mathrm{CE}$, they tended to veer toward the direction of the impairment.

\section{B. Muscle synergies and spinal maps during $O P$}

Extracted synergies were related to the control of the backward and the forward movements of the inverted pendulum by means of, respectively, TA and calf muscles, and the control of the trunk by means of the ES. Noticeably, the contribution of ankle plantarflexors and dorsiflexors during OE trials loaded two different synergies, highlighting a sort of decoupling of their roles while maintaning upright stance. The contribution of the other muscle groups was generally negligible and variable across subjects.
In the CE OP condition, we observed an anti-phase coupling of TA and GL. Specifically, they loaded the same synergy (often S1) with opposite sign (i.e., anti-phase coupling). Figure 2 shows a representative data set.

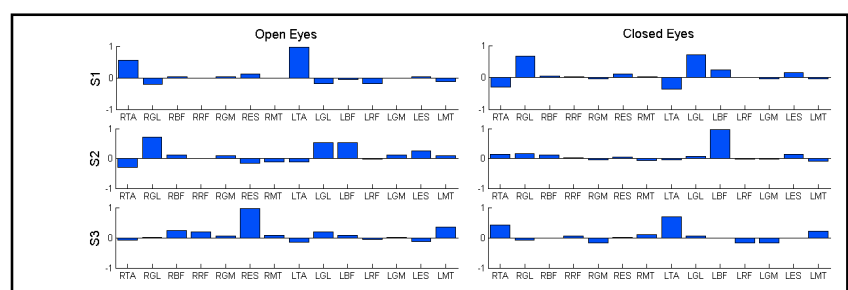

Figure 2. Representative set of weighting coefficients from a patient with left side vestibular disease during Orthostatic Posturography (OP) with OE (on the left) and CE (on the right). The prefix " $L$ " or " $R$ " of each $x$-axis tick respectively refer to the side Left and Right. The $y$-axis provide the value of the load for each muscle for retained synergies (i.e., S1, S2, and S3).

During OP with OE, we observed an asymmetric behavior of the spinal maps. In particular, the unaffected side was characterized by a bursts of activity at L2-L4 while the affected one showed a main burst at slightly caudal segments (L4-S1). When subjects were asked to close their eyes, the distal focus related to the affected side tended to shift toward most caudal sections (S1-S2) whereas the MN activity of the unaffected side mainly increased in amplitude. Data related to a representative patient are shown in Figure 3.

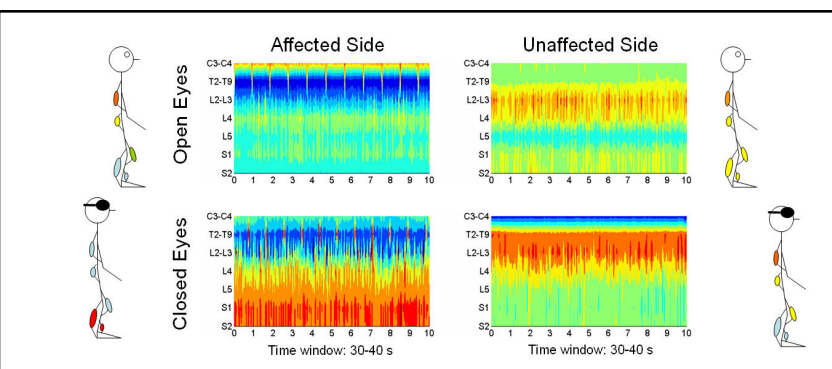

Figure 3. Representative spinal maps for a vestibular patient during Orthostatic Posturography (OP). The top and bottom subplots respectively refer to $\mathrm{OE}$ and $\mathrm{CE}$ trials. On both sides, representative human figures are drawn to illustrate the relationship between MN burts and muscle activity. The horizontal axis refers to the 30-40 s time window. The vertical axis refer to the spinal segments. The color scale refers to the amplitude (i..e, dark blue is the minimum, vivid red is the

\section{Muscle synergies and spinal maps during WT}

Muscle synergies extracted during WT trials did not appear significantly different than those reported in previous papers $[5,8]$ and were not significantly affected by the presence of the visual input. In particular, it was possible to distinguish among three main functional synergies: loading response, propulsive, and heel strike factors. 
In contrast to muscle synergies, spinal maps appeared slightly modified by the absence of visual input. Specifically, the burst of activity related to the push off in both sides (see Figure 4, level S1-S2, at about the $40-50 \%$ of the gait cycle of the affected side and at about the $90-100 \%$ of the unaffected side), and the foot clearance of the affected side (see Figure 4, level L2-L3, at about the $65-75 \%$ of the gait) were delayed reflecting the lengthening of the stance phase. Moreover, the affected side also showed a further burst at L2-L3 level highlighting the significant activity of knee extensors.
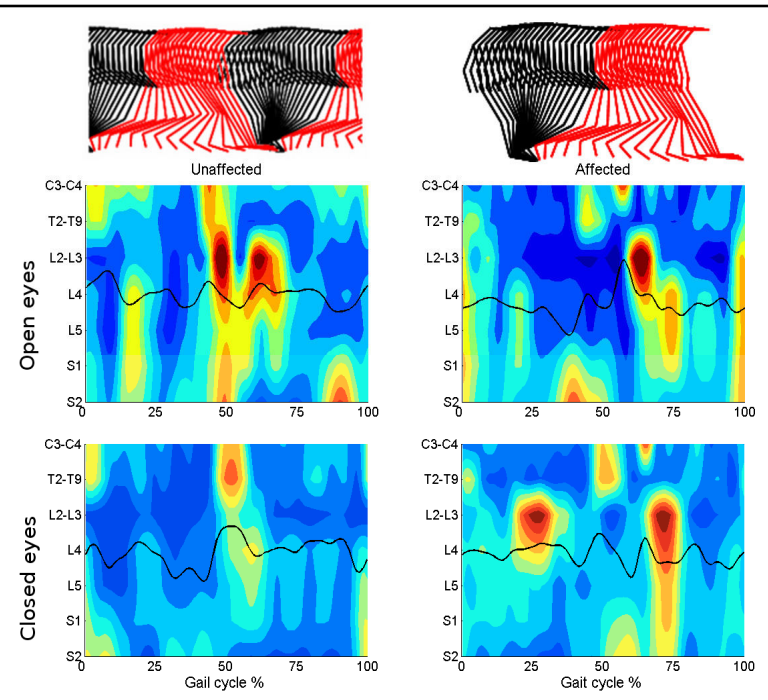

Figure 4. Representative spinal maps for a vestibular patient during Walking Trials (WT). The black line refer to the CoA. On the top, stick graphs of two gait cycles (black for the stance and red for the swing phase) are reported. Noticeably, the onset of all maps coincides with the heel strike of the affected side. Spinal maps on the top and the bottom respectively refer to $\mathrm{OE}$ and $\mathrm{CE}$ trials. The vertical axis refer to the spinal segments. The color scale refers to the amplitude (i..e, dark blue is the minimum, vivid red is the maximum).

\section{DISCUSSION}

This study aimed at highlighting the effect of visual feedback on the activity of $\mathrm{MN}$ pools belonging to the spinal cord between sections $\mathrm{C} 4$ and $\mathrm{S} 2$.

Concerning WT, the main result concerns the shift of some MN burst leading to a lengthening of the gait cycle with eyes closed. This result may reflect a more cautious behavior of vestibular patients while carrying out dynamic motor tasks.

Concerning the unperturbed standing, the expected result was that, when patients maintained upright stance with $\mathrm{CE}$, they used a more expensive balance control than during $\mathrm{OE}$ trials. It was documented by the greater amplitude of the statokinesigram (Figure 1 and Table II) reflecting a more intense action of the muscular system while balancing the inverted pendulum.
Results from spinal maps showed a shift in the focus of the MN activity toward caudal spinal sections of the affected side with absent vision (Figure 3). Accordingly, muscle synergies showed that patients tended to couple the activity of muscle groups crossing the ankle in the same synergy while carrying out OP with closed eyes (Figure 2). Actually, the postural control during the quite upright stance is achieved by ankle and hip strategies: the former involves rapid, presumably feedback-based, control movements; the latter involves slower, presumably feedforward-based, control movements. Results hence suggest that the absence of visual feedback involves a shift of the focus of the balance control toward distal muscles. This behavior is supposed to document an increased activity of sensory-motor afferences leading a more intense role of the forward based mechanism underlying balance control.

Further investigations are required to support these preliminary findings.

\section{REFERENCES}

[1] T. Brandt and M. Dieterich, "Postural imbalance in peripheral and central vestibular disorders," in Clinical disorders of balance posture and gait, T. B. A.M. Bronstein, M. Woollacott. Arnol Edition, Ed., 1996.

[2] J. L. Campos, J. S. Butler, and H. H. Bulthoff, "Multisensory integration in the estimation of walked distances," Exp Brain Res, Mar 13.

[3] J. J. Buchanan and F. B. Horak, "Voluntary control of postural equilibrium patterns," Behav Brain Res, vol. 143, pp. 121-40, Aug 14 2003.

[4] Y. P. Ivanenko, R. E. Poppele, and F. Lacquaniti, "Spinal cord maps of spatiotemporal alpha-motoneuron activation in humans walking at different speeds," J Neurophysiol, vol. 95, pp. 602-18, Feb 2006.

[5] V. Monaco, A. Ghionzoli, and S. Micera, "Age-related modifications of muscle synergies and spinal cord activity during locomotion," $J$ Neurophysiol, vol. 104, pp. 2092-102, Oct 2010.

[6] T. E. Prieto, J. B. Myklebust, R. G. Hoffmann, E. G. Lovett, and B. M. Myklebust, "Measures of postural steadiness: differences between healthy young and elderly adults," IEEE Trans Biomed Eng, vol. 43, pp. 956-66, Sep 1996.

[7] L. Quagliarella, N. Sasanelli, V. Monaco, G. Belgiovine, A. Spinarelli, A. Notarnicola, L. Moretti, and B. Moretti, "Relevance of orthostatic posturography for clinical evaluation of hip and knee joint arthroplasty patients," Gait Posture, vol. 34, pp. 49-54, May 2011.

[8] Y. P. Ivanenko, R. E. Poppele, and F. Lacquaniti, "Five basic muscle activation patterns account for muscle activity during human locomotion," J Physiol, vol. 556, pp. 267-82, Apr 12004. 